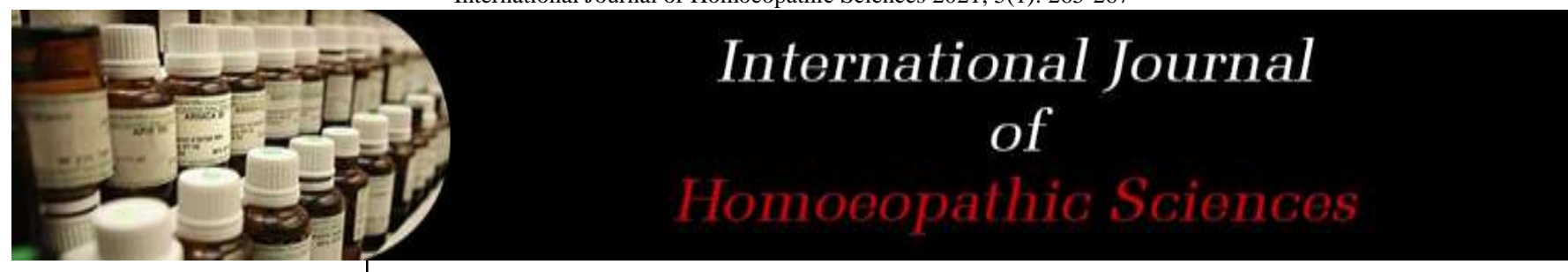

E-ISSN: $2616-4493$ P-ISSN: 2616-4485 www.homoeopathicjournal.com IJHS 2021; 5(1): 265-267 Received: $02-12-2020$ Accepted: 12-01-2021

Dr. Putchala Bhagya Vineesha PG Part 1, Department of Homoeo Pharmacy, Guru Mishri Homoeopathic Medical College, Maharashtra, India

Dr. Kavitha Khandare HOD, PG Guide, Guru Mishri Homoeopathic Medical College, Maharashtra, India

Dr. Dhanshree Joshi Guru Mishri Homoeopathic Medical College, Maharashtra, India
Corresponding Author: Dr. Putchala Bhagya Vineesha PG Part 1, Department of Homoeo Pharmacy, Guru Mishri Homoeopathic Medical College, Maharashtra, India

\section{Vitiligo and its homoeopathic management}

\author{
Dr. Putchala Bhagya Vineesha, Dr. Kavitha Khandare and Dr. \\ Dhanshree Joshi
}

DOI: https://doi.org/10.33545/26164485.2021.v5.i1e.323

\begin{abstract}
Vitiligo is an acquired dyschromia of the skin in which there is a loss of epidermal melanocytes. The prevalence of vitiligo is approximately $1 \%$ in the United States and $0.1-2 \%$ worldwide. The exact pathogenesis of vitiligo remains elusive and is likely multifactorial. After completing this update, participants should be able to discuss the epidemiology of vitiligo and summarize the proposed mechanisms for development of this disease. In addition, they should be able to discuss physical findings, approach to the patient, and some of the therapeutic modalities for this disorder.
\end{abstract}

Keywords: Homoeopathic management, Vitiligo, epidermal melanocytes

\section{Introduction}

Vitiligo is an acquired skin disorder caused by the disappearance of pigment cells from the epidermis that gives rise to well define white patches which are often symmetrically distributed. The lack of melanin pigment makes the lesional skin more sensitive to sunburn. Vitiligo occurs worldwide, with a prevalence of $\leq 2 \%$. In the United States, its estimated incidence is $1 \%$. The disease usually begins in childhood or young adulthood with a peak onset at 10-30 years. All races are affected, and both sexes are equally affected; however, a female preponderance has been reported. This discrepancy may potentially be skewed by increased reporting of cosmetic concerns by female patients. Vitiligo can be psychologically devastating for the affected patient. This is especially true in patients with darker skin because of the great contrast between the color of vitiliginous skin and surrounding normal skin. Vitiligo can affect quality of life, self-esteem, marriage, and employment, especially in darker-skinned individuals and in certain cultures because of confusion with leprosy and other contagious skin diseases. Loss of pigment may be viewed by patients as a threat to racial identity.

\section{Etiopathogenesis}

Vitiligo is multifactorial and polygenic. The precise pathogenesis remains elusive; however, several theories have been proposed to explain the loss of epidermal melanocytes in this disorder. Proposed mechanisms fall under the rubrics of autoimmune, biochemical, oxidantantioxidant, neural, and viral. Studies have also pointed to a significant role of genetic susceptibility to vitiligo.

\section{Genetics of Vitiligo}

Vitiligo is inherited in a non-Mendelian pattern and is characterized by incomplete penetrance, multiple susceptibility loci, and genetic heterogeneity. Inheritance may involve genes associated with melanin biosynthesis, response to oxidative stress, and regulation of autoimmunity. Because of the frequent association of vitiligo with autoimmune diseases, there have been investigations of possible HLA associations in vitiligo. Several haplotypes have been associated with vitiligo in more than one study.

The catalase gene has been implicated in the pathogenesis of vitiligo. Most likely a $\mathrm{C} / \mathrm{T}$ single nucleotide polymorphism in exon 9 of the catalase gene is responsible. Reduced catalase enzyme activity has been demonstrated in the epidermis of lesional and non-lesional skin in patients with vitiligo. Catalase is a peroxisomal enzyme found in nearly all organisms exposed to oxygen that catalyzes the decomposition of hydrogen peroxide to water and oxygen. Therein, it serves to prevent cell damage by highly reactive oxygen radicals.

\section{Role of the immune system}

The association of vitiligo with autoimmune conditions is well established. Thyroid disorders 
particularly Hashimoto's thyroiditis and Graves' disease, are commonly associated with vitiligo, as are other endocrinopathies, such as Addison's disease and diabetes mellitus. Alopecia areata, pernicious anemia, systemic lupus erythematosus, inflammatory bowel disease, rheumatoid arthritis, psoriasis, and autoimmune polyglandular syndrome also are associated, though the significance of some of these associations is debated. The most compelling argument for an autoimmune pathogenesis is the demonstration of circulating autoantibodies to melanocytes in the serum of patients with vitiligo. Autoantibodies directed specifically against melanocyte cell surface antigens have the ability to kill melanocytes in vivo and in vitro.

The levels of these autoantibodies seem to correlate with disease extent and activity. One of the autoantigens identified is VIT 40. Tyrosinase and TRP-1 and 2 have been identified as autoantigens as well, but data supporting this claim have been conflicting. Some patients with vitiligo have antibodies to melan A/MART-1, a melanocyte differentiation antigen. SOX transcription factors, which are involved in the differentiation of tissue derived from the neural crest, have been identified as melanocytic antigens in vitiligo associated with the polyendocrine syndrome. Antiorgan antibodies, such as antibodies to thyroglobulin, thyroid microsomes, and gastric parietal cells, also are frequently elevated in patients with vitiligo as compared with healthy control patients. Speculation abounds that codon-54 polymorphism in the mannose-binding lectin 2 gene may play a role in susceptibility to vitiligo. Mannosebinding lectin is a calcium-dependent lectin that causes predisposition to infections and autoimmune diseases.

\section{Oxidant-antioxidant role in vitiligo}

Oxidative stress may also play an important pathogenic role in vitiligo. Several studies suggest that accumulation of free radicals toxic to melanocytes leads to their destruction. Cultured melanocytes and the serum of patients with vitiligo often have increased nitric oxide levels, suggesting that nitric oxide could lead to autodestruction of melanocytes. Compared with control patients, the red cells of vitiligo patients have lower levels of glutathione, which helps prevent free radical mediated injury. Thus, vitiligo patients may be subject to a greater level of oxidative stress.

\section{Neural theory}

Segmental vitiligo often occurs in a dermatomal pattern. This observation led to a neural hypothesis that proposes certain chemical mediators released from nerve endings may cause decreased melanin production. Elevated neuropeptide Y levels have been demonstrated in skin affected by vitiligo. Decreased sweating occurs in some patches of segmental vitiligo, and some patients have been shown to have mild degenerative or regenerative changes in axons and Schwann cells in the depigmented areas.

\section{Viral}

Cytomegalovirus (CMV) DNA has been identified in skin biopsy specimens of some patients with vitiligo, which raises the question of whether there is viral-induced damage to melanocytes in subsets of patients with vitiligo. The possible involvement of other viruses, such as hepatitis $\mathrm{C}$, HIV, and Epstein-Barr virus has been suggested by some authors.

\section{Convergence theory}

Although all the aforementioned hypotheses are attractive, it is likely that vitiligo is a result of the convergence of several of these pathologic pathways. Most experts agree that vitiligo may indeed be a syndrome rather than a single entity.

\section{Classification of vitiligo}

Vitiligo is classified as segmental, acrofacial, generalized, and universal or by pattern of involvement as focal, mixed, and mucosal types.

- Focal vitiligo is usually a solitary macule or a few scattered macules in 1 area, most commonly in the distribution of the trigeminal nerve, although the neck and trunk are also commonly involved. This form occurs more commonly in children.

- Segmental vitiligo presents as unilateral macules in a dermatomal or quasi-dermatomal distribution. This type tends to have an early age of onset and, unlike the other types, is not associated with thyroid disease or other autoimmune diseases. Alteration of neural peptides has been implicated in the pathogenesis. More than one half of patients with segmental vitiligo have poliosis.

- Acrofacial vitiligo presents as depigmentation of the distal fingers and periorificial areas.

- Generalized vitiligo is also termed vitiligo vulgaris. This is the most common pattern. Depigmented patches are widely and usually symmetrically distributed.

- Universal vitiligo presents as depigmented macules and patches over most of the body and can be associated with multiple endocrinopathy syndrome.

Mucosal vitiligo involves only the mucous membranes.

\section{Clinical variants}

Trichrome vitiligo is characterized by both depigmented and hypo-pigmented macules in addition to normally pigmented skin. The natural evolution of the hypo-pigmented areas is progression to full depigmentation. Quadrichrome vitiligo refers to the additional presence of marginal or perifollicular hyperpigmentation. This variant is recognized more frequently in darker skin types, particularly in areas of repigmentation. Pentachrome vitiligo has also been reported with additional blue-gray hyper-pigmented macules, representing areas of melanin incontinence. Occasionally, patients with vitiligo may present with an unusual variant called the conVitiligo update 87 fetti type. These patients have several tiny, discrete, hypomelanotic macules. Inflammatory vitiligo is characterized clinically by erythema at the margins of vitiligo macules.

\section{Screening laboratory tests in patients with vitiligo \\ - Thyroid-stimulating hormone \\ - Complete blood count \\ - Total IG E count \\ - Antinuclear antibody \\ - Antithyroglobulin antibodies* \\ - Antithyroid peroxidase antibodies*}

*If patient and/or family history indicates.

\section{Management}

Vitiligo can be cosmetically disfiguring and it is a stigmatizing condition, leading to serious psychologic problems in daily life. It occurs worldwide in about $0.5 \%$ of the population and it occurs as frequently in males as it does in females. The cause is unknown, but might involve genetic 
factors, autoimmunity, neurologic factors, toxic metabolites, and lack of melanocyte growth factors. Since a causative (gene) treatment is not (yet) available, current modalities are directed towards stopping progression and to achieving repigmentation in order to repair the morphology and functional deficiencies of the depigmented skin areas.

Many treatments have been used for some time; however; there are some new developments: narrowband ultraviolet (UV) B $(311 \mathrm{~nm})$ therapy, the combination of corticosteroid cream + UVA therapy, and the transplantation of autologous pigment cells in various modalities. In widespread vitiligo, residual pigment can be removed by depigmentation agents. Sunscreens, camouflage products, and good guidance may help the patient cope better with the disease.

\section{Homoeopathic therapeutics}

Homeopathy has given innumerable successful results in the treatment of vitiligo. Homeopathy helps by correcting the auto-immune disorder and restoring the normal immune system. It helps in stimulating the melanocytes and boosts the production of the pigment melanin, thus restoring the normal skin colour.

Homeopathic prescription is based on the deepest level of psychological understanding of the patient thereby delving into the root cause of the issue. This individualized approach widens the scope of homeopathy even more.

- Ars Sulph Flavum: One of the rare, yet excellent remedies used to treat vitiligo in dry skin which may show cracks. Scaly, itchy, white spots on body parts are indicative of this remedy.

- Hydrocotyle Asiatica: Stimulates pigmentation of the skin in discoloured patches. Pricking on different parts of the skin.

- Ammi visnaga: It is indicated when patient has loss of pigmentation which is seen as white spots on skin. It has phototherapeutic properties which is very similar to psoralens. It is seen in some patients that use of A.visnaga along with exposure to sun causes repigmentation.

- Calc Carb: Wonderful medicine for milky white spots on skin in patients who are fair, fat, flabby and who tend to perspire a lot.

- Kali Carb: Dryness of skin with light-coloured spots all over the body. Sensation of burning and lancinating on the skin.

These are just some examples of the varied nature of remedies and symptoms of vitiligo. Treatment should be taken only under an experienced homeopath after a proper case taking and analysis. Homeopathy, when practiced with its principles, offers excellent results in vitiligo.

\section{Conclusion}

Vitiligo can be a very psychologically devastating disease. This is especially true in patients with skin of colour. Vitiligo carries with it myriad psychosocial implications. In dealing with vitiligo patients, it may be of use to take a multidisciplinary approach and involve psychology or psychiatry consultation early in the treatment course. Treatments for vitiligo have various response rates and it may be necessary to use rotational therapy to diminish side effects and to gain better re-pigmentation response.

\section{References}

1. Vitiligo Update Rebat Halder M, MD, Johnathan Chappell L. MD-Seminars in Cutaneous Medicine and Surgery Elsevier 2009;28:86-92.

2. David Njoo M. Wiete Westerh of - American journal of clinical dermatology 2001;2:167-181.

3. Halder R, Taliaferro Vitiligo S, In Wolff K, Goldsmith L, Katz S, Gilchrest B, Paller A, Lefell D. (eds) Fitzpatrick's Dermatology in General Medicine. New York, McGraw-Hill 2008;1(7).

4. Halder R, Nandedkar M, Neal K. Pigmentary disorders in pigmented skins, In Halder R (ed): Dermatology and Dermatological Therapy of Pigmented Skin. New York, NY, Informa Healthcare 2005;5(1).

5. Gavalas NG, Akhtar S, Gawkrodger DJ et al. Analysis of allelic variants in the catalase gene in patients with the skin depigmenting disorder vitiligo. Biochem Biophys Res Commun 2006;345:1586-1591.

6. Casp CB, She JX, McCormack WT. Genetic association of the catalase gene (CAT) with vitiligo susceptibility. Pigment Cell Res 2002;15:62-66.

7. Bystryn JC. Immune mechanisms in vitiligo. Clin Dermatol, Vitiligo update 91 1997;15:853-861.

8. Palermo B, Campanelli R, Garbelli S et al. Specific cytotoxic $\mathrm{T}$ lymphocyte responses against Melan A/Mart1, tyrosinase and gp 100 in vitiligo by the use of major histocompatability complex/peptide tetramers: The role of cellular immunity in the etiopathogenesis of vitiligo. J Invest Dermatol 2001;117:326-332.

9. Ongenae K, Van Geel N, Naeyaert J. Evidence for an autoimmune pathogenesis of vitiligo. Pigment Cell Res 16:90-100. Adi BS. Efficacy of homoeopathic medicines in chronic low back pain: a clinical study. International Journal of Alternative and Complementary Medicine 2020, P17-20.

10. Onay H, Pehlivan M, Alper S et al. Might there be a link between mannose binding lectin and vitiligo? Eur J Dermatol 2007;17:146-148.

11. Hazneci E, Karabulut AB, Oztürk C et al. A comparative study of superoxide dismutase, catalase, and glutathione peroxidase activities and nitrate levels in vitiligo patients. Int J Dermatol 2005;44:636-640.

12. Tu C, Zhao D, Lin X. Levels of neuropeptide $Y$ in the plasma and skin tissue fluids of patients with vitiligo. J Dermatol Sci 2001;27:178-182.

13. Grimes PE, Sevall JS, Vojdani A. Cytomegalovirus DNA identified in skin biopsy specimens of patients with vitiligo. J Am Acad Dermatol 1996;35:21-26.

14. Akbayir N, Gukdemir G, Mansur T et al. Is there any relationship between hep $\mathrm{C}$ virus and vitiligo? J Clin Gastroenterol 2004;38:815-817.

15. Niamba P, Traore A, Taieb A. Vitiligo in a black patient associated with HIV infection and repigmentation under antiretroviral therapy. Ann Dermatolo Venereol 2007;134:272-273. 